\title{
In Vivo Spectroscopy vis- à -vis Ex Vivo Spectroscopy: Pearls and Pitfalls: Imaging Review
}

Khizer Razak, Surbhi Gupta and Meena GL*

Department of Radiodiagnosis, S.P. Medical College, Bikaner, Rajasthan, India

*Corresponding author: Meena GL, Department of Radiodiagnosis, S.P. Medical College, Bikaner, Rajasthan, India, Tel: +919413143709; E-mail: meenabkn@yahoo.co.in

Received date: November 15, 2017; Accepted date: November 27, 2017; Published date: December 05, 2017

Copyright: (c) 2017 Razak K, et al. This is an open-access article distributed under the terms of the Creative Commons Attribution License, which permits unrestricted use, distribution, and reproduction in any medium, provided the original author and source are credited.

\begin{abstract}
Aims and objective: The objective of this communication is to bring the metabolomics by magnetic resonance (high resolution spectroscopy) to the clinical radiologist and compare the spectroscopy in vivo (NMR spectroscopy used in clinical) with the high resolution spectroscopy ex vivo (magic angle spectroscopy, HR -MORE). S and analyzing differences, advantages and limitations of each technique in different tissues.

Review of the topic: In vivo spectroscopy that is usually performed in the clinic is a non-invasive technique that can provide important information for the diagnosis and monitoring of different lesions. The most well-known applications of in vivo spectroscopy today are those carried out by localized $1 \mathrm{H}$ spectroscopy, monovoxel or multivoxel (spectroscopic image), brain, prostate or breast. There are other modalities, such as the $31 \mathrm{P}$ or $13^{\circ} \mathrm{C}$ spectroscopy, but they are much less frequent and are only carried out in a few specialized centres. Without any doubt, the most widespread clinical use is confined to the central nervous system.

Conclusion: The in vivo spectroscopy provides important metabolic information for the diagnosis of different pathologies, non-invasively, however, this technique has certain limitations, such as low spectral resolution, that is, the number of metabolites that can be identified/quantified; which results in low diagnostic specificity.

Ex vivo spectroscopy, however, has a spectral resolution and sensitivity far superior to in vivo techniques, which allows detecting a large number of metabolites, and therefore greatly improves its diagnostic capacity. However, we must not forget that it is an invasive technique, since it is based on the analysis of pieces of tissue (biopsies, surgical pieces...) and therefore loses its usefulness in those lesions that are impossible to biopsy. In conclusion, each technique has its added value and its limitations and it is the correct use of them and the combination of them with other diagnostic parameters, where their clinical potential resides.
\end{abstract}

Keywords: Spectroscopy; Imaging; Peak; Cerebral; Diagnosis; Prostate

\section{Introduction}

The high-resolution spectroscopy used in metabolomics studies is a technique in which high-field vertical spectrometers (11-23 T) are used for the analysis of biofluids or small pieces of intact tissue (for example biopsies), obtaining spectroscopic results of multiple metabolites that are not visible by in vivo spectroscopy [1].

In this communication we will focus on the technique that analyzes intact tissue, known as HR-MAS, from the English high resolution magic angle spinning (Figure 1).

HR-MAS spectroscopy has much higher sensitivity and resolution than in vivo spectroscopy, which allows determining the metabolic profile or "metabolic fingerprint" of a tissue sample of between 5 and $20 \mathrm{mg}$. The spectral resolution is comparable to that obtained in liquid spectroscopy, from tissue extracts, with the great advantage that it does not require any manipulation of the sample and therefore the tissue can be recovered and reused later for diagnosis in pathological anatomy (Figure 2).
In vivo spectroscopy that is usually performed in the clinic is a noninvasive technique that can provide important information for the diagnosis and monitoring of different lesions.
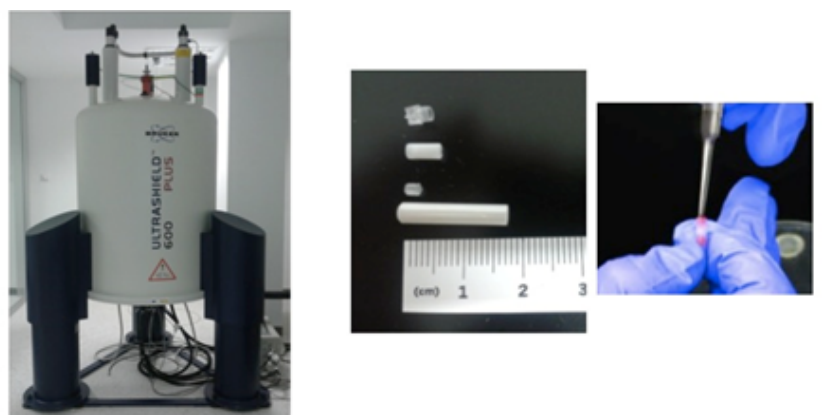

Figure 1: $600 \mathrm{MHz}(14 \mathrm{~T})$ high resolution vertical spectrometer, with HR-MAS probe (left); zirconium rotor and insert in which the piece of tissue is placed for its analysis by HR-MAS (right). 


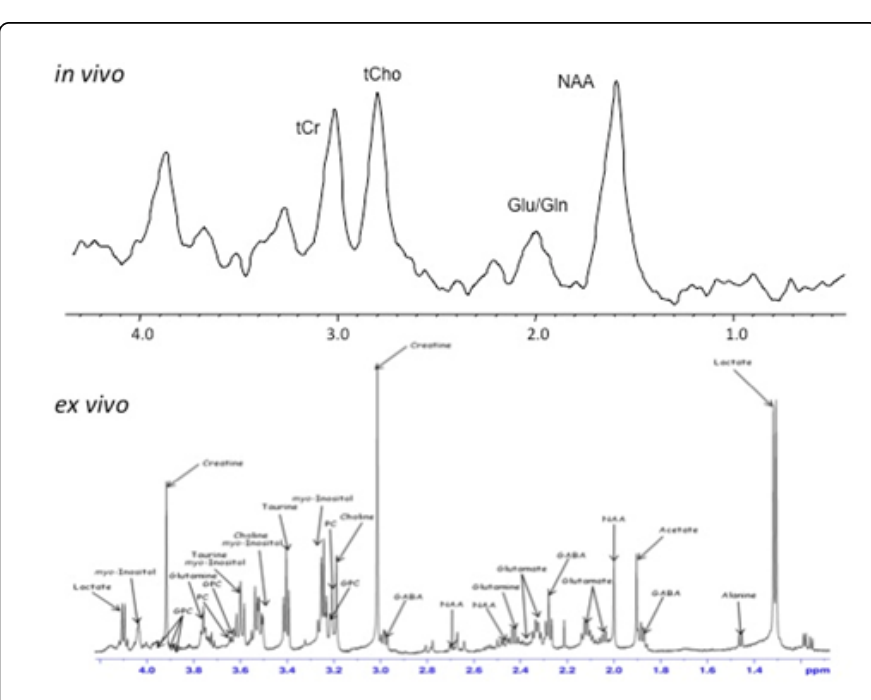

Figure 2: Ex vivo HR-MAS spectroscopy has great sensitivity and resolution compared to in vivo spectroscopy, which allows determining the metabolic fingerprint of an intact tissue sample, while in vivo spectroscopy only it allows to solve a few metabolites.

The most well-known applications of in vivo spectroscopy today are those carried out by localized $1 \mathrm{H}$ spectroscopy, monovoxel or multivoxel (spectroscopic image), brain, prostate or breast. There are other modalities, such as the $31 \mathrm{P}$ or $13 \mathrm{C}$ spectroscopy, but they are much less frequent and are only carried out in a few specialized centres.

Currently, ex vivo spectroscopy is not implemented in hospitals, but its research results promise an interesting future for the diagnosis and management of patients.

\section{Background and Discussion}

Without any doubt, the most widespread clinical use of spectroscopy confined to the central nervous system. The main metabolites used in diagnosis in the CNS are shown in Figure 3.

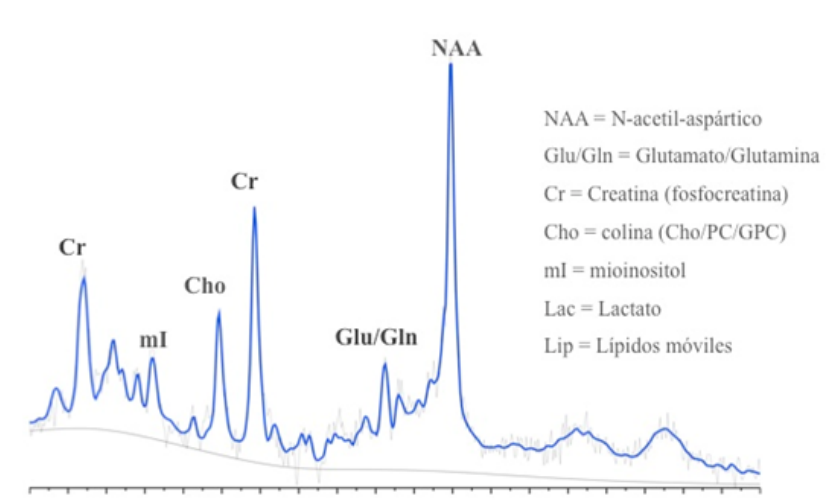

Figure 3: $1 \mathrm{H}$ monovoxel spectrum in vivo of healthy brain tissue in which the main metabolites that can be observed in this type of studies are indicated.
NAA (N-Acetyl Aspartic)- Neuronal Marker. Its decrease reflects a neuroaxonal impoverishment (typical in glial tumors).

$\mathrm{mI}$ (myoinositol)- Glial marker Its increase reflects an increase in the glial contribution [in glial tumors it is indicative of low grade tumor]. Its decrease indicates glial de-differentiation (high-grade tumors)

Cho (Colina)- Membrane Metabolism. In Tumors, it is a Proliferative Activity Indicator. Increases in demyelination and inflammatory processes

Cr (Creatine)- Marker of Energy Metabolism. It is typically diminished in malignant tumors (high grade)

Mobile lipids- Malignancy marker (necrosis). It is not usually talk about malignancy unless they are present. They also appear with radiotherapy treatment (radionecrosis).

Lac (Lactate)- Marker of Anaerobic Metabolism. Typical of active tumors against quiescent tumors. It also appears in hypoxic/ischemic and infectious/inflammatory processes.

Regarding the prostate, the number of metabolites observed is lower:

Citrate- Marker of prostatic glandular tissue without alterations. It drastically decreases in adenocarcinomas.

Cho (Hill) - Proliferative activity marker. It increases in adenocarcinomas.

Polyamines. Like citrate, its presence is an indicator of normal glandular metabolism and is diminished in adenocarcinomas.

Finally, in the breast, given the large contribution of lipid signals, which make it difficult to observe most metabolites in vivo, the only metabolite used for tumor diagnosis is choline, which rises significantly in tumors.

Spectroscopy by HR-MAS ex vivo on its side has the advantage of having a high sensitivity and spectral resolution which allows the detection of multiple metabolites not visible by spectroscopy in vivo, therefore, the diagnostic specificity is much higher. For example, in vivo spectroscopy only resolves a peak in the area of choline-derived metabolites, while HR-MAS allows its different components to be separated: Phosphocholine (PC) Glycerophosphocholine (GPC) and Choline (Cho) (Figures 4 and 5).

Bibliographic review of the results of in vivo spectroscopy compared to high resolution spectroscopy ex vivo and the contribution in the diagnosis of different pathologies of both techniques, in particular in three tumor types: cerebral, prostate and breast.

\section{Cerebral}

In vivo spectroscopy: In vivo spectroscopy at the brain level has shown its usefulness in tumor evaluation, for which several metabolites have been measured: NAA, Cho, lactate, creatine and myoinositol (Figure 3)

Cho levels increase in a multitude of tumors, but at the brain level, their levels are significantly higher when the tumor is present, therefore they are useful for differentiating gliomas of low, high grade and benign cerebral pathology. 
NAA (N-Acetyl Aspartic acid) decreases in those pathologies in which there is neuroaxonal impoverishment, as occurs in glial tumors [2].

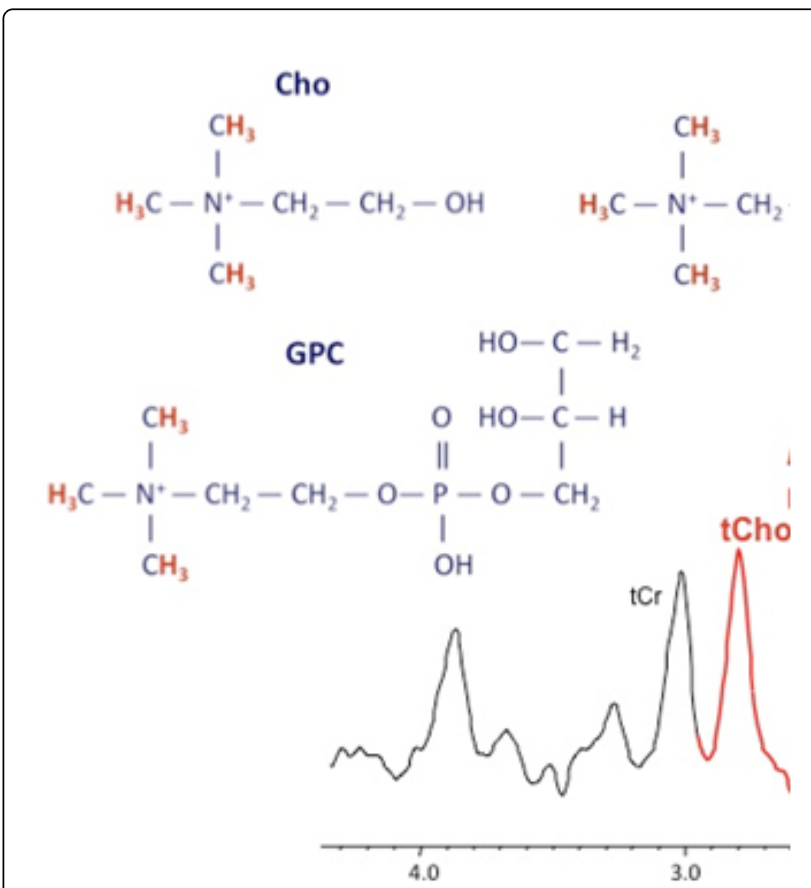

Figure 4: Brain spectrum of $1 \mathrm{H}$ in vivo in which the total choline peak (tCho) is shown, composed essentially of three metabolites, choline, phosphocholine and glycerol phosphocholine, which cannot be differentiated in vivo.

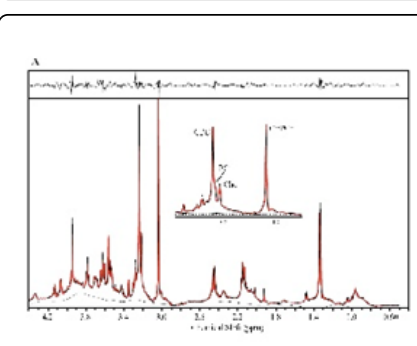

Low Grade Glioma

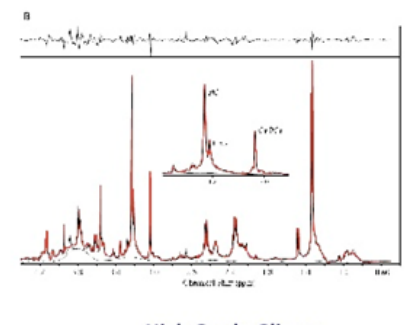

High Grade Glioma
Figure 5: $1 \mathrm{H}$ HR-MAS spectra (high resolution magic angle spinning) of glial tumor biopsies showing how the highest spectral resolution allows us to differentiate the contributing individual components from the total Choline peak (tCho): choline, phosphocholine and glycerophosphocholine. The relationships between these three metabolites allow the differentiation of low and high grade gliomas.

These metabolites have been used to differentiate low-grade gliomas with ischemic lesions in young people without risk factors for cerebral infarction, where the diagnosis by MRI is less complicated, thus, lowgrade gliomas present high peaks of Cho (choline), absence of NAA (N acetyl aspartate) and undetectable Lactate, while infarcts present low peaks of Cho, NAA and a significant increase in lactate. Lactate has been increased in high-grade gliomas, but these usually do not present difficulty in their diagnosis in the image [2].

Ex vivo spectroscopy: The HR-MAS at the brain level is able to quantify a multitude of metabolites that are not visible by conventional spectroscopy in vivo.

Martinez-Bisbal et al. performed HR-MAS spectrum of high-grade glioma biopsies, determining 37 metabolites [3].

On the other hand it has been shown that there is a good correlation of in vivo and ex vivo diagnosis with the only exception that metabolites related to anaerobic metabolism, lactate and alanine are increased in ex vivo, due to the period of hypoxia experienced by tissue pieces until they are frozen [3].

In a study published in 2009, it was determined that the different levels of the components of the peak of total choline (tCho), GPC (glycerophosphocholine), PC (phosphocoline) and Cho (free Choline) and the relationship between these, is different according to whether of gliomas of low or high grade. Thus, in low-grade gliomas, the predominant component is CPG whereas in high-grade gliomas PC predominates [4] (Figure 5).

On the other hand, there seems to be a correlation between certain metabolites detected by HR-MAS and tumor grading. Thus, the increase in the PC/GPC ratio is characterized by severe forms of glioblastoma multiforme. And an increase in the ratio of myoinositol/Cho allows differentiating the clinical evolution of different low-grade gliomas [5].

The usefulness of HR-MAS for the differential diagnosis between aggression of meningiomas has also been demonstrated, it was determined that the glutamine and glutamate metabolites that are related to the metabolism of glutathione are significantly more increased in more aggressive meningiomas and with greater possibility therefore of recurrence [6].

\section{Prostate}

In vivo spectroscopy: In the prostate, in vivo spectroscopy using endorectal MRI with multivoxel technique has proved very useful for the diagnosis of prostate cancer. The prostatic epithelial cells have the peculiarity of producing a large amount of citrate under normal conditions, as a consequence of an inhibition of the zinc-mediated enzyme aconitase. However, when tumor transformation occurs, this inhibition is lost and citrate levels fall drastically (Figure 6). On the other hand, as in other tumor processes, choline increases significantly.

This technique has proved to be very useful in cases of elevated PSA to locate areas of tumor suspicion and thus direct biopsy to a specific area (Figure 7). On the other hand, in cases of radiotherapy treatment, prostate spectroscopy is very useful for the diagnosis of local recurrence, since after the treatment the prostate tissue presents an anodyne appearance and the imaging techniques are not very informative. However, spectroscopy allows observing the peak of choline in those areas where there is recurrence [7].

Ex vivo spectroscopy: The determination of ex vivo metabolites that are not resolved by in vivo spectroscopy has provided relevant information in prostate cancer. Thus, it has been determined that malignant and non-malignant tissue can be differentiated using the ratios of the GPC (Glycerophosphocholine)+PC (phosphocholine) and Creatine spectra. 

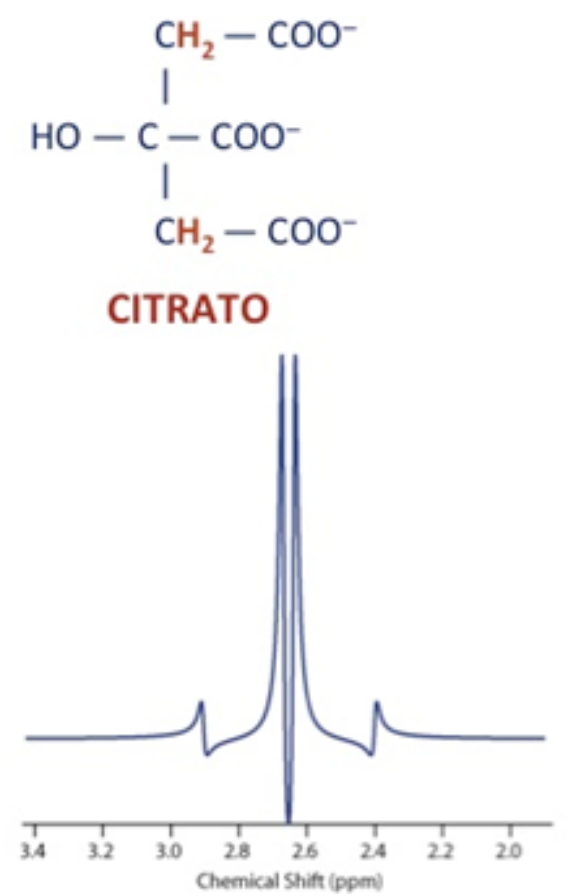

\begin{tabular}{|l|l|}
$\begin{array}{l}\text { Citrate levels in Human Prostate Gland (nmoles) } \\
g)\end{array}$ \\
\hline Central Zone & 5.000 \\
\hline Peripheral Zone & 13.000 \\
\hline Prostatic Fluid & $40.000-150.000$ \\
\hline BPH (mixed tissue) & $10.000-15.000$ \\
\hline BPH (glandular tissue) & $20.000-50.000$ \\
\hline PC (mixed tissue) & $1.000-3.000$ \\
\hline PC (malignant tissue) & $<500$ \\
\hline Stromal Tissue & $150-300$ \\
\hline Other soft tissues & $150-450$ \\
\hline Blood Plasma & $90-110$ \\
\hline
\end{tabular}

Figure 6: Citrate signal as observed in vivo at $1.5 \mathrm{~T}$ and table of concentration in different tissues.

In addition it has been determined that hills derivatives are better tumor biomarkers than citrate itself, especially in the central prostate, since citrate appears high in prostatic epithelial cells and low in tumor, but also appears low in stroma. However, the hills appear high only in tumor tissue (twenty).

On the other hand it has been seen that the Citrate/Creatine relationship is significantly related to tumor aggressiveness (twenty). Finally, it is interesting to mention the work of Asten et al. in which it is demonstrated that the prostate biopsy tissue is not altered after the analysis by HR-MAS, so it can be used for histological diagnosis [8].

\section{Breast}

In vivo spectroscopy: The elevation of the choline peak, as in other types of cancer, is used as a biomarker of tumor activity, however, caution should be used with this statement since, as has been shown in several publications, the elevation of choline may be due to also to other pathologies of benign character, in particular fibroadenomas, tubular adenomas and during lactation [9-12].

On the other hand, the quantification of this metabolite by in vivo spectroscopy has shown its usefulness in determining the response to treatment before clinical response data appear. Thus, there are studies that indicate that $24 \mathrm{H}$ after treatment with doxorubicin, there are significant changes of the choline peak in the tumor lesion and that the decrease in the peak at $24 \mathrm{H}$ is associated with a better response to treatment $[3-5,7,10,11,13-18]$.

$E_{X}$ vivo spectroscopy: As in other tissues, ex vivo spectroscopy allows the analysis of many non-visible metabolites by in vivo spectroscopy, which raises the possibility of establishing a diagnosis of tumor aggressiveness based on the metabolic profile or metabolome. This is especially attractive in the case of the breast

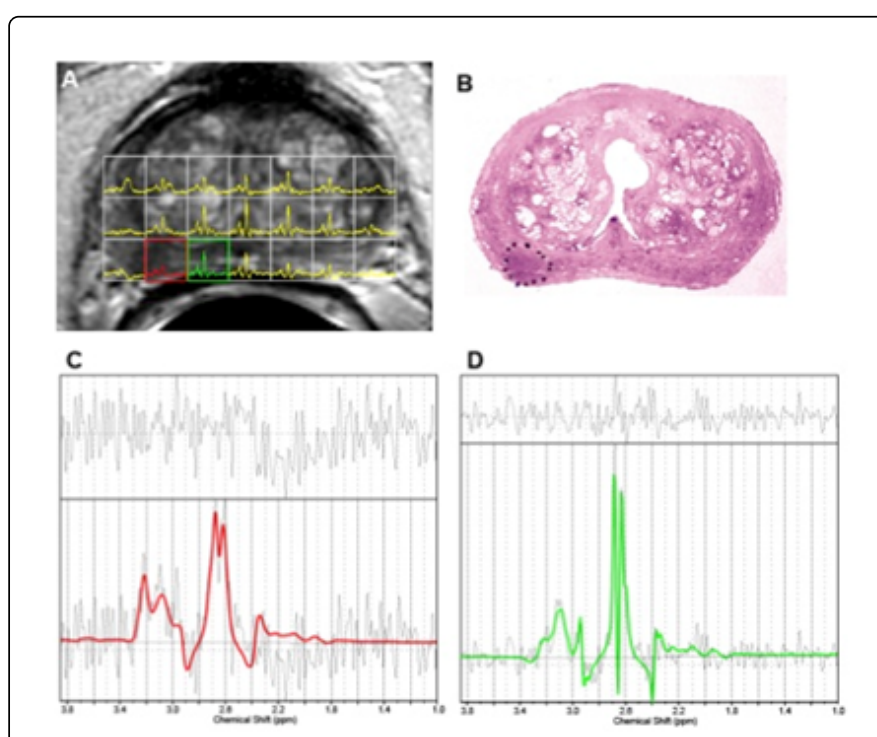

Figure 7: Spectroscopic image (or multivoxel spectroscopy) of prostate in vivo. The spectrum corresponding to the tumor zone (red) shows an increase of the choline peak and decrease of citrate compared to the spectrum of a healthy zone (green). The histological section is also shown in which it is confirmed that the alteration of the metabolic profile corresponds to a tumor nodule.

because in vivo spectroscopy poses great difficulties due to the abundance of lipids in the breast tissue, which make it extremely difficult to carry out routinely (Figure 8). 


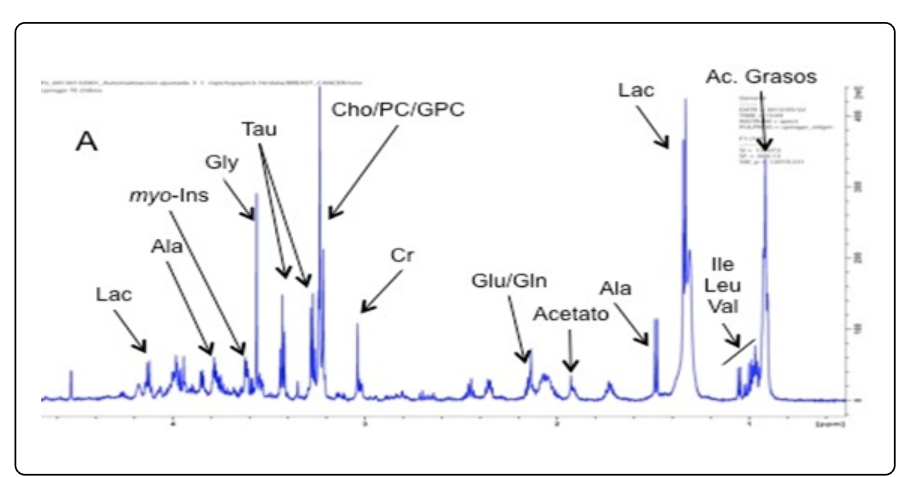

Figure 8: 1H HR-MAS spectrum of a breast cancer biopsy. Unlike in vivo spectroscopy that only allows lipids and choline to be seen, this technique allows obtaining the complete metabolic profile of the biopsy.

It has been shown that there is a metabolomic pattern of breast cancer, characterized mainly by increased lactate and glycine, and PC with respect to GPC [14]. Comparing the metabolomic profile with clinical parameters, it has been shown that Glycine, Taurine and PC are higher in tumors of higher degree and that the PC/GPC ratio is very increased in tumor tissue with respect to healthy [15].

In another study, it was detected that high levels of glycine and lactate predicted significantly lower 5 year survival in cancers with SR (positive estrogen receptors), whereas this relationship did not occur in patients with negative ER. The 5 year survival prediction results in women with ER positive cancers were more robust than the prediction made clinically [16].

In the models with the best prognosis [basal-like], the GPC concentration is higher than the PC concentration and the opposite occurs in the luminal-like $[17,19]$. The repercussion of a possible parallelism between metabolic profile and genetic type supposes an important field for the metabolomics, since the genotypic studies of breast cancer have a high cost, its realization requires a lot of time and also the realization of these involve the destruction of the samples.

\section{Conclusion}

The in vivo spectroscopy provides important metabolic information for the diagnosis of different pathologies, non-invasively, however, this technique has certain limitations, such as low spectral resolution, that is, the number of metabolites that can be identified/quantified; which results in low diagnostic specificity.

EX vivo spectroscopy, however, has a spectral resolution and sensitivity far superior to in vivo techniques, which allows detecting a large number of metabolites, and therefore greatly improves its diagnostic capacity. However, we must not forget that it is an invasive technique, since it is based on the analysis of pieces of tissue (biopsies, surgical pieces...) and therefore loses its usefulness in those lesions that are impossible to biopsy.

The results obtained in the studies published for the metabolomic study of breast cancer promise a very prosperous future for this type of techniques with a possible application in clinical settings.

On the other hand, there is an important connection with the radiological diagnosis since it is a spectroscopic study of the tissues, something that the radiologist already does in his day to day in the study by in vivo NMR $[20,21]$. On the other hand, this technique has great advantages with respect to its rapid realization without tissue destruction, which could be analyzed anatomopathologically later, so it is not risky to think that the HR-MAS diagnosis is applied in a clinical setting in a short time and that be the radiologist in charge of its interpretation.

For its final translation to the clinic it is necessary to improve the spectral analysis system, so that it is automatic, robust and precise. With regard to breast cancer, it has been shown that there is a metabolomic pattern of breast cancer: increased lactate, glycine and PC (phosphocholine) with respect to GPC (glycerophosphocholine). The case of the typical pattern of the hills is significantly elevated in cases of positive ER (Estrogen Receptor), and in the determination of involvement of surgical borders, as well as in mouse models for cancer with luminal-like pattern, which raises a diagnosis of tumor aggressiveness of a metabolic spectrum.

Especially interesting is the possibility of metabolomic analysis of breast biopsies that would be performed in a short time and without tissue destruction. As we have commented, the studies carried out so far with breast biopsies have not been very solid, so further studies are needed to determine the diagnostic, evolutionary and subsequent management impact of each radiological pattern.

In conclusion, each technique has its added value and its limitations, and it is the correct use of them and the combination of them with other diagnostic parameters, where their clinical potential resides.

\section{References}

1. Righi V, Roda JM, Paz J, Mucci A, Tugnoli V, et al. (2009) 1H HR-MAS and genomic analysis of human tumor biopsies discriminate between high and low grade astrocytomas. NMR Biomed 6: 629-637.

2. Soaresa DP, Lawb M (2009) Magnetic resonance spectroscopy of the brain: Review of metabolites and clinical applications. Clin Radiol 64: 12-21.

3. Martinez-Bisbal MC, Monleon D, Assemat O, Piotto M, Piquer J, et al. (2009) Determination of metabolite concentrations in human brain tumour biopsy samples using HR-MAS and ERETIC measurements. NMR Biomed 22: 199-206.

4. Comet Batlle J, Vilanova JC, Barceló Obregón J, Maroto Genover A, Osorio Fernández M, (2006) Diagnóstico del cáncer de próstata mediante espectroscopía de Resonancia Magnética endorectal. Arch Esp Urol 10: 953-963.

5. Elkhaled A, Jalbert L, Constantin A, Yoshihara HA, Phillips JJ (2014) Characterization of metabolites in infiltrating gliomas using $e x$ vivo $1 \mathrm{H}$ high-resolution magic angle spinning spectroscopy. NMR Biomed 27: 578-593.

6. Gonzalez-Darder J, Talamantes F, Cortés O, Gil-Benson R, López-Gines C, et al. (2008) Benign and atypical meningioma metabolic signatures by high-resolution magic-angle spinning molecular profiling. J Proteome Res 7: 2882-2888.

7. Borgan E, Lindholm EM, Moestue S, Mælandsmo GM, Lingjærde OC, et al. (2013) Subtype-specific response to bevacizumab is reflected in the metabolome and transcriptome of breast cancer xenografts. Mol Oncol 7: 130-142.

8. Asten J, Cuijpers V, Hulsbergen-van de Kaa C (2008) High resolution magic angle spinning NMR spectroscopy for metabolic assessment of cancer presence and Gleason score in human prostate needle biopsies. Magn Reson Mater Phy 21: 435-442.

9. Bathen TF, Geurts B, Sitter B, Fjøsne HE, Lundgren S, et al. (2013) Feasibility of MR metabolomics for immediate analysis of resection margins during breast cancer surgery. PLoS ONE 8: 61578. 
Citation: Razak K, Gupta S, Meena GL (2017) In Vivo Spectroscopy vis-à-vis Ex Vivo Spectroscopy: Pearls and Pitfalls: Imaging Review. Mol

10. Bolan PJ, Nelson MT, Yee D, Garwood M (2005) Imaging in breast cancer: Magnetic resonance spectroscopy. Breast Cancer Res 7:149-152.

11. Giskeødegård GF, Lundgren S, Sitter B, Fjøsne HE, Postma G (2012) Lactate and glycine-potential MR biomarkers of prognosis in estrogen receptor-positive breast cancers. NMR Biomed 25:1271-1279.

12. Cecil KM, Schnall MD, Siegelman ES, Lenkinski RE (2001) The evaluation of human breast lesions with magnetic resonance imaging and proton magnetic resonance spectroscopy. Breast Cancer Res Treat 68: 45-54.

13. Kvistad KA, Bakken IJ, Gribbestad IS, Ehrnholm B, Lundgren S, et al. (1999) Characterization of neoplastic and normal human breast tissues with in vivo $1 \mathrm{H}$ MR spectroscopy. J Magn Reson Imaging 10: 159-164.

14. Borgan E, Sitter B, Lingjærde OC, Johnsen H, Lundgren S (2010) Merging transcriptomics and metabolomics-advances in breast cancer profiling. BMC Cancer 10: 628 .

15. Bathen TF, Jensen LR, Sitter B, Fjösne HE, Halgunset J, et al. (2007) MRdetermined metabolic phenotype of breast cancer in prediction of lymphatic spread, grade and hormone status. Breast Cancer Res Treat 104: 181-189.

16. Sitter B, Lundgren S, Bathen TF, Halgunset J, Fjosne HE, et al. (2006) Comparison of HR MAS MR spectroscopic profiles of breast cancer tissue with clinical parameters. NMR Biomed19: 30-40.
17. Giskeødegård GF, Lundgren S, Sitter B, Fjøsne HE, Postma G (2012) Lactate and glycine-potential MR biomarkers of prognosis in estrogen receptor-positive breast cancers. NMR Biomed 25:1271-1279.

18. Grinde MT, Skrbo N, Moestue SA, Rødland EA, Borgan E,et al. (2014) Interplay of choline metabolites and genes in patient-derived breast cancer xenografts. Breast Cancer Res 16: 5 .

19. Jagannathan NR, Kumar M, Seenu V, Coshic O, Dwivedi SN, et al. (2001) Evaluation of total choline from in vivo volume localized proton MR spectroscopy and its response to neoadjuvant chemotherapy in locally advanced breast cancer. Br J Cancer 84: 1016-1022.

20. Moestue SA, Borgan E, Huuse EM, Lindholm EM, Sitter B, et al. (2010) Distinct choline metabolic profiles are associated with differences in gene expression for basal-like and luminal-like breast cancer xenograft models. BMC Cancer 10: 433.

21. Stenman K, P.är Stattin, Stenlund H, Riklund K, Gröbner G (2011) $1 \mathrm{H}$ HRMAS NMR derived bio-markers related to tumor grade, tumor cell fraction and cell proliferation in prostate tissue samples. SAGE Journals 6. 